\title{
Acute triphenyltin intoxication: a case report
}

\author{
Ruey-Meei Wu, Yang-Chyuan Chang, Hou-Chang Chiu
}

\begin{abstract}
A 23 year old male developed abdominal pain, diarrhoea, and vomiting several hours after poisoning with acute triphenyltin intoxication in a suicide attempt. Severe ataxia, dysmetria, nystagmus, and blurring of vision soon supervened. Disturbance of consciousness and confusion developed 12 days later and lasted for two months. A delayed sensorimotor polyneuropathy was shown by electrophysiological studies to be due to axonal degeneration and demyelination. The neuropathy rapidly recovered after consciousness was regained.
\end{abstract}

Organotins are widely used in agricultural practice for their bactericidal, antiparasitic, fungicidal and molluscicidal properties. The neurotoxicity of triethyltin and trimethyltin has already been recognised in both human cases and animal studies. ${ }^{1-4}$ Triphenyltin (TPhT) has been regarded as less neurotoxic than trialkyltins. ${ }^{56}$ Only a few patients with mild TPhT poisoning have been reported. ${ }^{7}$ We report a case with acute severe $\mathrm{TPhT}$ intoxication, characterised by severe encephalopathy and delayed polyneuropathy.

\section{Case report}

A 23 year old male developed abdominal pain, diarrhoea, and vomiting on 13 October 1986. Headache, low grade fever, blurred vision, and unsteady gait occurred the next day. A lumbar puncture revealed normal cerebrospinal fluid (CSF) findings. Because of neurological deterioration, he was transferred to the National Taiwan University Hospital on 21 October.

On admission, the patient was conscious but restless. Blood pressure was $110 / 70$ $\mathrm{mm} \mathrm{Hg}$, pulse was $92 / \mathrm{min}$ with occasional ventricular premature contractions and temperature was $38 \cdot 5^{\circ} \mathrm{C}$. There was no neck stiffness. Moderate impairment of recent memory, abstract thinking, and calculation was found. Cranial nerves were intact. He had a severe ataxic gait and prominent cerebellar dysfunction. Muscle power and tendon reflexes were normal. Sensations were intact. An EEG on 22 October revealed poorly organised alpha rhythms with intermittent generalised paroxysmal bursts of high voltage delta waves (fig 1a). Brainstem auditory evoked potentials (BAEP) were normal. Cranial CT showed no abnormal findings. A lumbar puncture the next day still failed to disclose abnormal findings.

The patient became confused and occasionally semicomatous after the fifth day in hospital. A follow up EEG on 18 November revealed a moderate slowing of background activity (4-6 Hz) with periodic paroxysmal generalised spikes or sharp waves, recurring every 1-2 seconds (fig 1b). A lack of definite diagnosis indicated preference for conservative treatment only.

The patient gradually regained consciousness two months after admission. In late December 1986, a crucial piece of information was obtained: the patient had attempted suicide using a molluscicidal agent. Mass spectrometric analysis confirmed this agent to be triphenyltin compound. In early January 1987 the patient began to improve. There was no more nystagmus. Moderate weakness in the lower limbs with mild wasting in the small foot muscles was found; the tendon reflexes were absent in the lower limbs and hypoactive in the upper. A detailed evaluation of sensory function was impossible. Motor conduction velocities were reduced: median nerve in the left $45.7 \mathrm{~ms}$, ulnar nerve $45.2 \mathrm{~ms}$, peroneal nerve $29.9 \mathrm{~ms}$, posterior tibial nerve $33.7 \mathrm{~ms}$. Sensory conduction velocities were also slowed: left median nerve $46.8 \mathrm{~ms}$, ulnar nerve $37.8 \mathrm{~ms}$, sural nerve $32.6 \mathrm{~ms}$. Motor and sensory action potential (MAP and SAP) amplitudes were severely decreased: peroneal nerve MAP $0.5 \mathrm{mV}$, posterior tibial nerve MAP $4.5 \mathrm{mV}$, and sural nerve SAP $5 \mathrm{uV}$. Electromyography of the small muscles of the
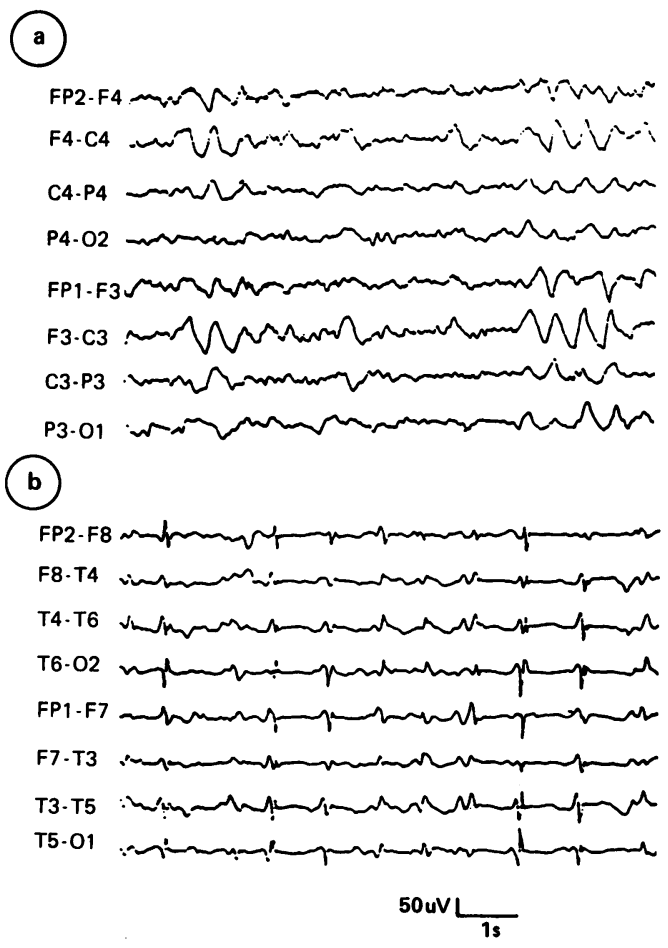

Figure 1 EEG after TPhT intoxication: (a) on day 10 showing poorly organised alpha rhythms with intermittent generalised bursts of high voltage delta waves; and (b) on day 40 showing quasiperiodic paroxysmal generalised spikes recurring in 1-2 s. 
hand and foot showed active fibrillation and positive waves. The patterned visual evoked potential showed prolonged P100 latencies with low amplitude. Only Wave V with prolonged latency was recognisable in the BAEP. Somatosensory evoked potentials by stimulation of the median or peroneal nerves revealed prolonged absolute latencies and central conduction times.

The patient continued to improve rapidly. In Febreuary 1987, he regained consciousness. However, mild memory impairment and occasional disinhibited behaviours were noted. Muscle power was much improved. His tendon reflexes became hypoactive in the lower limbs and normal in the upper. Sensations of all modalities were mildly impaired with a glove-stocking pattern. An EEG obtained 3.5 months after the poison was taken revealed well organised alpha rhythms with rare generalised spikes and rare bifrontal delta bursts. A follow up neurography showed significant recovery of NCVs, MAP amplitudes, and SAP amplitudes. There was also a parallel improvement of all evoked potential abnormalities.

In addition to neurological symptoms, the patient had inappropriate antidiuretic hormone secretion, upper gastrointestinal bleeding, mildly elevated serum transaminase levels, leukopenia with WBC counts fluctuating from 2500 to $5000 /$ cumm, and myeloid hypoplasia in the bone marrow.

\section{Discussion}

Clinical features of the 1954 TET-intoxicated French patients included intracranial hypertension, paralysis, and generalised convulsions. ${ }^{12}$ Acute TMT intoxication has resulted in a limbic-cerebellar syndrome in six industrial workers. ${ }^{4}$ Their clinical features were hearing loss, disorientation, confabulation, amnesia, aggressiveness, hyperphagia, disturbed sexual behaviour, seizures, nystagmus, ataxia, and mild sensory neuropathy. Mild TPhT intoxication from occupational exposure caused nausea, vomiting, photophobia, impaired vision, headache, vertigo, weakness, lower limb paresthaesia, convulsions, and transient loss of consciousness. ${ }^{7}$ All of these features disappeared within two weeks. The early features in the present case were abdominal pain, diarrhoea, headache, vomiting, visual impairment, and severe cerebellar dysfunctions. Consciousness disturbance developed 12 days later. Finally, polyneuropathy was found 2.5 months after poisoning. Because our patient had attempted suicide, he had a more severe neurological involvement.

In human TET cases and in experimental animals, diffuse intramyelinic oedema was the main necropsy finding. ${ }^{12}$ The major pathological change in a TMT fatal case was neuronal alterations in the limbic system structures and cerebellar Purkinje's cells, ${ }^{4}$ similar to the condition found in TMTtreated rats. No significant pathological change was found in the central nervous system of TPhT-fed animals. No human necropsy has been reported.

Acute toxic symptoms in TPhT-fed animals were watery diarrhoea, anorexia, weakness, staggering gait, paralysis of the hind limbs, tremor, convulsion, and death due to coma. ${ }^{6}$ A number of biochemical changes have been observed in animals, including reduced glucose utilisation, ${ }^{7}$ catecholamine depletion, ${ }^{7}$ inhibition of microsomal adenosine triphosphatase, ${ }^{6}$ reduction of aldehyde dehydrogenase activity, ${ }^{6}$ inhibition of mitochondrial oxidative phosphorylation, ${ }^{6}$ and inhibition of Na-K-ATPase in cell membranes. ${ }^{6}$ The same biochemical mechanisms might also be responsible for acute TPhTinduced encephalopathy in humans.

Peripheral nerve involvement in TPhT intoxication has not been well described, ${ }^{7}$ but lower limb parathesias in the previous human cases and paralysis in the hind limbs of guinea pigs $^{6}$ were evidence of the existence of TPhTinduced polyneuropathy. Electrophysiologically, the marked amplitude reduction of action potentials and prominent electromyographic abnormality in our case indicated unequivocal axonopathic changes in the peripheral nerves. However, a profound slowing of nerve conduction velocities in the lower limbs and rapid recovery of the patient's polyneuropathic manifestations suggest the important role of demyelination in the pathogenesis of TPhT-induced nerve damage.

Hearing impairment, a common feature in human TMT poisoning, ${ }^{4}$ was also seen in our case. Detailed audiometry studies were not done in this case, but BAEP examinations revealed normal findings in the beginning, loss of early waves with a prolonged $\mathrm{V}$ wave latency 2.5 months later, and a return to normal of the BAEP waveforms before discharge. These BAEP findings might indicate reversible ototoxicity in TPhT intoxication.

$\mathrm{TPhT}$-induced liver damage, such as transient elevation of serum transaminase levels in our case, has been confirmed in previous human cases $^{7}$ and various animal species. ${ }^{6}$ Bone marrow toxicity has never been reported in human victims, but Bock reported significant leukopenia in TPhT-fed animals. ${ }^{6}$

Different organotins cause different toxic syndromes. Reasons for such a discrepancy are worthy of further investigation.

We thank Miss Hong-Ping Li, Residue Control Department, Taiwan Agricultural Chemicals and Toxic Substances Research Institute, for analysis of the toxic substance.

1 Watanabe I. Organotin. In: Spencer PS, Schaumburg $\mathbf{H H}$ eds. Experimental and clinical neurotoxicology. Baltimore: Williams and Wilkins, 1980:545-56.

2 Foncin JF, Gruner JE. Tin neurotoxicity. In: Vinken PJ, Bruyn GW, eds. Handbook of Clinical Neurology, Vol 36. Bruyn GW, eds. Handbook of Clinical Neur
Amsterdam: North-Holland, 1979:279-90.

3 Graham DI, V de Jesus P, Pleasure DE, Gonatas NK. Triethyltin sulfate-induced neuropathy in rats. Arch Neurol 1976;33:40-8.

4 Besser R, Kramer G, Thumler R, Bohl J, Gutmann L, Hopf HC. Acute trimethyltin limbic-cerebellar syndrome. Neurology 1987;37:945-50.

5 Snoeij NJ, Van Iersel AAJ, Penninks AH, Seinen W. Toxicity of triorganotin compounds: comparative in vivo studies with trialkyltin compounds and triphenyltin chloride in male rats. Toxicol Applied Pharmacol 1985;81:274-86.

6 Bock R. Triphenyltin compounds and their degradation products. In: Gunther FA, ed. Residue reviews, vol 79. New York: Springer-Verlag, 1981:31-57.

7 Manzo L, Richelmi P, Sabbioni E, Pietra R, Bono F, Guardia $L$. Poisoning by triphenyltin acetate. Report of Guardia L. Poisoning by triphenyltin acetate. Report of two cases and determination of tin in blood and urine by 